\title{
Seroprevalence of Dengue Viral Infection among Adults Attending the University of Cape Coast Hospital
}

\author{
Ebenezer Aniakwaa-Bonsu', Daniel Amoako-Sakyi', Kwabena Dankwa1, \\ James Kojo Prah², Samuel Victor Nuvor ${ }^{1 *}$ \\ ${ }^{1}$ Department of Microbiology and Immunology, School of Medical Sciences, University of Cape Coast, Cape Coast, Ghana \\ ${ }^{2}$ Directorate of University Health Services, University of Cape Coast, Cape Coast, Ghana \\ Email: *s.v.nuvor@uccsms.edu.gh
}

How to cite this paper: Aniakwaa-Bonsu, E., Amoako-Sakyi, D., Dankwa, K., Prah, J.K. and Nuvor, S.V. (2021) Seroprevalence of Dengue Viral Infection among Adults Attending the University of Cape Coast Hospital. Advances in Infectious Diseases, $11,60-72$.

https://doi.org/10.4236/aid.2021.111008

Received: February 6, 2021

Accepted: March 19, 2021

Published: March 22, 2021

Copyright $\odot 2021$ by author(s) and Scientific Research Publishing Inc. This work is licensed under the Creative Commons Attribution International License (CC BY 4.0).

http://creativecommons.org/licenses/by/4.0/

\begin{abstract}
Introduction: Most febrile illnesses in Ghana are often misdiagnosed and presumptively treated as malaria. This situation may be due to the inappropriate diagnostic tool, clinical oversight and lack of awareness of some of the disease conditions that might have been present in the country. This study sought to investigate the seroprevalence of dengue virus, geographical location of participants with circulating antibodies and finally evaluate the diagnostic accuracy of a Rapid diagnostic kit (RDT) using Enzyme Linked Immuno-Sorbent Assay (ELISA) as a gold standard for confirmation. Method: A hospital-based cross-sectional study was conducted among adults $(\geq 18)$ attending the University of Cape Coast Hospital. From each participant, 3 - 4 $\mathrm{ml}$ of venous blood was drawn and serum was tested for IgG and IgM using RDT methods. Positive samples were selected for ELISA confirmation. Statistical analysis was performed using SPSS (v.20) and STATA (v.14) software. Results: A total of 270 participants were enrolled in the study. The geometric mean age was 32 years. Overall, Dengue virus (DENV) IgG seroprevalence by RDT was 10 (3.7\%). Seroprevalence of IgG and IgM by the ELISA method was $34(12.6 \%)$ and $6(2.2 \%)$ respectively. Females recorded a high seroprevalence $(7.4 \%)$ than males $(5.2 \%)$ in terms of past exposure (IgG). On current exposure (IgM), females recorded a high seroprevalence (1.5\%) than males (0.7\%). Seroprevalence of individuals with dual positivity $((\operatorname{IgG}+\operatorname{IgM}+)$ (acute secondary infection) was $1.9 \%$. Those with primary and recent infection (IgM+ only) were $0.4 \%$ whiles those with past and probably secondary infection (IgG+ only) were $10.7 \%$. The odds of dengue exposure were significantly high among participants with ages 50 - 59 as compared to their other counterparts $(\mathrm{AOR}=3.5, \mathrm{p}=0.03$ ). Diagnostic accuracy of RDT kit was poor
\end{abstract}


(Kappa value $=0.373$ and 0.0001$)$. Elmina was noted to record the highest seroprevalence values for both anti-dengue IgG (3.3\%) and IgM (1.85\%). Conclusion: The seroprevalence of anti-dengue IgG and IgM among participants suggests that dengue virus is in circulation in Cape Coast Metropolis and Komenda Edina Eguafo Abirem Municipality.

\section{Keywords}

Seroprevalence, ELISA, RDT, Dengue Virus

\section{Introduction}

Dengue virus (DENV) infection is an evolving health threat that is now assuming considerable global public health concern. Since 2012, it has been shown to be the most serious arboviral disease transmitted by mosquitoes globally [1] with greater morbidity and economic impact [2]. Data from the World Health Organization suggested that an estimated number of 2.5 billion people dwell in endemic areas [3] and over 50 million dengue infection occur annually [4]. Generally, the infection has been reported in the tropics and sub-tropics of the globe with an estimated 19,000 related deaths recorded in 2002 [4]. In non-endemic areas, the bulk of notified cases of the infection were mostly spread by international travelers that had visited dengue-endemic areas [4]. Seroprevalence surveys had revealed dengue exposure in sub-Saharan Africa [5] with West Africa as the probable region for the viral transmission due to the presence of the transmitting vector, urbanization, poor sanitary measures and inadequate diagnostic tool [6].

Though there are no official records on the dengue virus outbreak in Ghana, few studies done have confirmed the presence of dengue infection and its serotypes [7]. A work done by Amoako et al., (2018) in Ghana recorded a seroprevalence figure of $21.5 \%$. Again, another work done in Ghana to detect dengue among 150 patients with suspected Ebola virus disease showed that $15 \%$ tested positive for both IgG and IgM, 4 for non-structural protein 1 (NS1) whiles 4 tested positive for viral RNA [8]. In a study of 605 feverish children in Ghana, only $11 \%$ tested positive for malaria by microscopy after $80 \%$ had been misdiagnosed with malaria [9]. This data suggest that the etiology of febrile illness goes beyond Plasmodium infections.

The signs of dengue fever typically mimic febrile illnesses such as malaria, hence, it is presumptively treated as malaria in most endemic countries [10]. There is therefore potential risk for dengue outbreak as several serotypes may be circulating among human population putting them at risk of antibody-dependent enhancement (ADE) dengue haemorrhagic fever (DHF) and dengue syndrome shock (DSS) if there is secondary infection with a heterologous serotype. There is, therefore, the need to do routine and differential diagnosis of dengue so there can be a laboratory evidence-based treatment. This will aid in the reduction of 
indiscriminate use of antimalarial and antibiotics and thereby reducing resistance in circulation.

This study therefore aims at determining the seroprevalence of dengue virus, geographical location of participants with circulating antibodies and finally evaluating the diagnostic accuracy of some commercial kits.

\section{Methods and Materials}

\subsection{Study Site}

This was a hospital-based cross-sectional study conducted at the University of Cape Coast Hospital in Cape Coast, Ghana. The facility provides various health care services through in-patient and out-patient departments. Cape Coast Metropolis has a double annual rainfall with a mean temperature of $25^{\circ} \mathrm{C}$ [11]. This kind of climate condition favours the breeding of the mosquito vector that transmits the virus causing the disease. Again, the transmitting vector thrives in areas with increasing urbanization, overcrowding and poor sanitation. The most common means of discarding refuse in Cape Coast is by either dumping in a container (56.7\%) or dumping unto an open dumpsite (21.1\%) [11]. In most dwelling places in the city, liquid waste is disposed of by throwing it onto the compound (34.0\%) [11]. The Metropolis recorded 66.7\% of houses in urban areas which served as a home to $75 \%$ of household population. Averagely, there were 2.3 households per house in Cape Coast as compared to a regional and national average of 1.5 and 1.6 respectively [11]. Finally, Cape Coast has tourist attraction sites hence attracts a large number of tourists globally and locally. This increases the vulnerability of the local residents to the infection since some of these travelers may be coming from hyperendemic areas that might have been exposed to the virus.

\subsection{Study Design}

The study was a cross-sectional hospital-based and the sampling method used was convenient sampling. Participants were selected without any randomization so far as they consented and qualify to be included in the study.

\subsection{Population}

The research was conducted among patients who were seeking routine medical care at inpatient and outpatient department of University of Cape Coast Hospital from February 2019 to July 2019. These patients included both males and females who were 18 years and above and at least presented with fever and three malaria-like symptoms. Participants were selected based on inclusion and exclusion criteria.

\section{Sample size}

The minimum sample size was determined by the formula (Cochran).

$$
S=Z^{2}(P)(1-P) /(\text { Error })^{2}
$$


where $Z=1.96$ as the standard normal variate at 5\% type error, $P=21 \%$

$$
\text { Minimum sample size, } \begin{aligned}
S & =1.96^{2}(0.21)(1-0.21) /(5 / 100)^{2} \\
& =254.9 \approx 255 \text { clinical sample }
\end{aligned}
$$

Using 5\% non-responsive rate, 270 participants were enrolled.

\subsection{Inclusion and Exclusion Criteria}

Individuals included in this study were 18 years and above presenting with fever and at least three symptoms of the following: myalgia, arthralgia, rash, headache, abdominal pain, retro-orbital or ocular pain and haemorrhagic manifestation (i.e. petechiae, purpura, epistaxis, gum bleeding, etc.). The study excluded patients who visited the health facility without presenting at least three of the symptoms mentioned above, people below age eighteen, and those who were not willing to partake in the study.

\subsection{Sample Collection and Storage}

The blood samples transported from the hospital to the laboratory were spun at $3000 \times \mathrm{g}$ for 5 minutes at $4^{\circ} \mathrm{C}$. A sterile disposable transfer pipette was used to transfer serum into a sterile $1.5 \mathrm{ml}$ Eppendorf tube and stored at $-20^{\circ} \mathrm{C}$ until used.

\subsection{Detection of IgG/IgM Using Rapid Diagnostic Test (One Step RDT)}

The rapid detection of anti-dengue IgG and IgM was done using One-step RDT kit (Voyage Medicals, China). For a positive result, three pink bands or two pink bands appeared on test region of the cassette indicating that the specimen contained detectable amount of anti-dengue IgG and IgM. A test was considered invalid if a coloured band did not appear at control region, indicating a possible error in performing the test, hence, test repeated.

\subsection{Detection of IgM Using ELISA}

All samples were brought to room temperature and tested for anti-dengue IgM using ELISA kits (DIA source Immunoassays S.A, Belgium). Briefly, $50 \mu \mathrm{l}$ of neutralising reagent was added to all wells except, the blank well A1 and the control wells which were added with positive and negative control samples in duplicate. Finally, $100 \mu \mathrm{l}$ of diluted samples were added to each well and the microplate incubated for 60 minutes at a temperature of $37^{\circ} \mathrm{C}$. The microwells were then washed five times and $100 \mathrm{ul}$ of enzyme conjugate were added to each well except the blank well and covered with a sealer. The microplate was incubated for another 60 minutes at $37^{\circ} \mathrm{C}$ and washed after which $100 \mu \mathrm{l}$ of the chromogenic substrate mixture was added to each well including the blank well. The microplate was then incubated at room temperature $\left(25^{\circ} \mathrm{C}\right)$ for 20 minutes in the dark and 100 ul of Sulphuric acid added for yellow colour development. The colour intensity of the solution in each well was measured at an optical density 
(OD) of $450 \mathrm{~nm}$ (blanking A1 well). The test results were interpreted as a ratio of sample OD450 $\mathrm{nm}$ value (S) and the Cut-Off value (Co) or S/Co. Ratio < 0.9 were considered negative, $0.9-1.1$ were considered equivocal and $>1.1$ were considered positive.

\subsection{Detection of IgG ELISA}

ELISA kits (DIA source Immunoassays S.A, Belgium) were used for determining IgG levels in the participant. Briefly, $10 \mu \mathrm{l}$ of diluted serum sample (1:100) was vortexed and added to each well together with $100 \mu \mathrm{l}$ of positive control and negative control. The microplate was then incubated for 60 minutes at a temperature of $37^{\circ} \mathrm{C}$ and washed five times. Hundred microliters (100 $\left.\mu \mathrm{l}\right)$ of enzyme conjugate were then added to each well except the blank well and covered with a sealer. The microplate was again incubated for 60 minutes at $37^{\circ} \mathrm{C}$, washed and $100 \mu \mathrm{l}$ of the chromogenic substrate mixture added including the blank well. The microplate was then incubated at room temperature $\left(25^{\circ} \mathrm{C}\right)$ for 20 minutes in the dark and Sulphuric acid $(100 \mu \mathrm{l})$ was added for the development of yellow colour. The colour intensity of each well solution was measured at an optical density (OD) of $450 \mathrm{~nm}$. Samples showing an OD $450 \mathrm{~nm}$ value lower than the Cut-Off value (blank wells) were considered negative for anti-dengue IgG while samples with OD 450 higher than the Cut-Off were considered as positive.

\subsection{Data Analysis}

Data was analyzed using STATA (version 14). Measures of central tendency, means, medians, frequency distribution and percentages were calculated. Binary logistic regression was used to assess the risk of dengue exposure for gender, age and type of settlement. Cohens Kappa Inter-rater reliability was also used to assess the performance characteristics of rapid diagnostic kit.

\subsection{Ethical Consideration}

The study received ethical approval from the University of Cape Coast Institutional Review Board (UCCIRB). Participation in the study was voluntary and informed consent was obtained from each participant.

\section{Results}

\subsection{Sociodemographic Characteristics}

General characteristics of the participants were analyzed to determine the frequency and percentage distributions in terms of age, gender, educational background, occupational status and the type of settlement. From the results analyzed (Table 1), the geometric mean age and its $95 \%$ CI was 32 (30.69-33.54). Majority of participants were 18 years to 28 years 106 (39.26\%). Few of them were 60 years and above $49(7.04 \%)$. In terms of gender distribution, out of 270 participants, 103 (38.1\%) were males and 167 (61.9\%) were females. Majority of the participants; 139 (51.48\%) had had tertiary education. Those with senior 
Table 1. Demographic data of the participants in the study.

\begin{tabular}{|c|c|c|}
\hline Variable & Geometric mean $(95 \% \mathrm{CI})$ & n (\%) \\
\hline Age & $32(30.69-33.54)$ & \\
\hline \multicolumn{3}{|l|}{ Age category } \\
\hline $18-28$ & & $106(39.26)$ \\
\hline $29-39$ & & $77(28.52)$ \\
\hline $40-49$ & & $33(12.22)$ \\
\hline $50-59$ & & $35(12.96)$ \\
\hline$\geq 60$ & & $19(7.04)$ \\
\hline \multicolumn{3}{|l|}{ Sex } \\
\hline Male & & $103(38.15)$ \\
\hline Female & & $167(61.85)$ \\
\hline \multicolumn{3}{|l|}{ Educational status } \\
\hline Non-formal & & $24(8.90)$ \\
\hline Primary & & $5(1.85)$ \\
\hline JHS & & $37(13.7)$ \\
\hline SHS/Votec & & $65(24.07)$ \\
\hline Tertiary & & $139(51.48)$ \\
\hline \multicolumn{3}{|l|}{ Occupational Status } \\
\hline Farmers & & $3(1.11)$ \\
\hline Students & & $83(30.74)$ \\
\hline Civil servants & & $84(31.11)$ \\
\hline Artisans & & $18(6.67)$ \\
\hline Health Professionals & & $6(2.22)$ \\
\hline Traders & & $62(22.96)$ \\
\hline Unemployed & & $14(5.19)$ \\
\hline \multicolumn{3}{|l|}{ Settlement type } \\
\hline Urban & & $139(51.48)$ \\
\hline Semi-Urban & & $131(48.52)$ \\
\hline
\end{tabular}

$\mathrm{N}=270$.

high school/vocational-technical were 65 (24.07\%). Participants with junior high school and primary education were $37(13.7 \%)$ and $5(1.85 \%)$ respectively. Finally, individuals with no formal education were $24(8.8 \%)$.

More than half $(139,51.48 \%)$ of the participants lived in semi-urban areas whiles 131 (48.52\%) lived in urban areas. Majority of the participants recruited in this study were civil servants: 84 (31.11\%).

\subsection{Seroprevalence}

The overall seroprevalence in this study was $12.96 \%$ (IgG and IgM combined). 
The seroprevalence for IgG was $12.6 \%$ whiles IgM was $2.2 \%$ (Table 2). The seropositivity rate was higher in females than males for both IgG and IgM. The age group with the highest IgG seropositivity rate were those between ages 29 and 39. This same group recorded the highest seroprevalence rate in terms of current exposure (IgM). Participants who were attending the University hospital from Elmina recorded the highest IgG (3.3\%) and IgM (1.85\%) seropositivity rate as compared to those from other towns. Apewosika also recorded an IgM seropositivity rate of $0.37 \%$. Apewosika and Elmina were the only towns where IgM antibodies were detected among participants.

The seroprevalence of participants with recent primary infection (IgG-IgM+) was $0.37 \%$ (Table 3 ). Those with recent secondary infection (IgG + IgM+) were $1.85 \%$. The seroprevalence of past primary infection (IgG + IgM-) or probable secondary infection was $10.74 \%$. Two hundred and thirty-five (235) (87.04\%) did not show any evidence of dengue infection (IgG-IgM-).

\subsection{Risk of Exposure}

The odds of dengue exposure in females was 0.12 ( $\mathrm{AOR}=0.88,95 \% \mathrm{CI}$ : 0.40 1.90) times lower than in their male counterparts (Table 4). However, the odds of dengue exposure in participants with ages 50 - 59 was significant with 3.5 ( $95 \%$ CI: $1.13-10.85, p=0.03)$ times higher than those between ages $18-28$. For

Table 2. Seropositivity rate according to gender, age and geographical location.

\begin{tabular}{|c|c|c|}
\hline Variable & $\operatorname{IgG}(\%)$ & $\operatorname{IgM}(\%)$ \\
\hline Overall & 12.6 & 2.2 \\
\hline \multicolumn{3}{|l|}{ Gender } \\
\hline male & 5.1 & 0.74 \\
\hline female & 7.41 & 1.48 \\
\hline \multicolumn{3}{|l|}{ Age } \\
\hline $18-28$ & 2.59 & \\
\hline $29-39$ & 4.07 & 1.11 \\
\hline $40-49$ & 2.59 & 0.74 \\
\hline $50-59$ & 2.22 & 0.37 \\
\hline$\geq 60$ & 1.11 & \\
\hline \multicolumn{3}{|l|}{ Location } \\
\hline Elmina & 3.3 & 1.85 \\
\hline Akotokyir & 0.37 & \\
\hline Abura & 1.48 & \\
\hline Kotokuraba & 1.85 & \\
\hline Apewosika & 1.9 & 0.37 \\
\hline UCC & 2.6 & \\
\hline Kingsway & 1.11 & \\
\hline
\end{tabular}


Table 3. Seropositivity rate of primary and secondary infection.

\begin{tabular}{|c|c|c|c|c|c|}
\hline $\begin{array}{l}\text { Serological } \\
\text { group }\end{array}$ & $\operatorname{IgM}$ & IgG & $\mathbf{N}$ & $\begin{array}{l}\text { Seropositivity } \\
\quad \text { rate }(\%)\end{array}$ & Interpretation \\
\hline 1 & + & - & 1 & 0.37 & Recent/primary infection \\
\hline 2 & + & + & 5 & 1.85 & Recent secondary infection \\
\hline 3 & - & + & 29 & 10.74 & $\begin{array}{c}\text { Past Primary infection/ } \\
\text { probable secondary infection }\end{array}$ \\
\hline 4 & - & - & 235 & 87.04 & No evidence of current/past exposure \\
\hline
\end{tabular}

Table 4. Univariate and Multivariate analysis of sex, age and settlement type as predictive factors for dengue infection.

\begin{tabular}{|c|c|c|c|c|c|c|}
\hline \multirow{3}{*}{ Variable } & \multicolumn{6}{|c|}{ IgG/IgM } \\
\hline & \multicolumn{3}{|c|}{ Multivariate } & \multicolumn{3}{|c|}{ Univariate } \\
\hline & AOR & $95 \% \mathrm{CI}$ & p-value & COR & $95 \% \mathrm{CI}$ & p-value \\
\hline \multicolumn{7}{|l|}{ Sex } \\
\hline males & ref. & & & ref. & & \\
\hline females & 0.88 & $0.40-1.90$ & 0.74 & 0.86 & $0.42-1.79$ & 0.698 \\
\hline \multicolumn{7}{|l|}{ Age } \\
\hline $18-28$ & ref. & & & ref. & & \\
\hline $29-39$ & 2.43 & $0.88-6.71$ & 0.085 & 2.36 & $0.81-5.45$ & 0.09 \\
\hline $40-49$ & 3.11 & $0.96-10.05$ & 0.06 & 3.14 & $0.94-9.51$ & 0.06 \\
\hline $50-59$ & 3.50 & $1.13-10.84$ & $0.03^{*}$ & 3.54 & $1.11-9.98$ & $0.03^{*}$ \\
\hline$\geq 60$ & 2.59 & $0.6-11.13$ & 0.20 & 2.65 & $0.58-10.85$ & 0.19 \\
\hline \multicolumn{7}{|l|}{ Settlement } \\
\hline Urban & ref. & & & ref. & & \\
\hline Semi-urban & 1.24 & $0.59-2.59$ & 0.561 & 1.22 & $0.59-2.52$ & 0.58 \\
\hline
\end{tabular}

the other age groups, the risk of dengue exposure was high as compared to the reference category (18 - 28) but there was no statistical evidence of association. The risk of infection was high among participants in semi-urban settlement than their counterparts in the urban settlement $(\mathrm{AOR}=1.24,95 \% \mathrm{CI}: 0.59-2.59)$.

\subsection{Performance Characteristics of Kit}

The results shown in Table 5 indicate the diagnostic parameters of RDT (One step, Voyage Medicals, China) using ELISA as a gold standard. This analysis was subjected to Cohen's Kappa inter-rater reliability statistical tool. The Cohn's Kappa coefficient value was 0.373 and 0.0001 for IgG and IgM respectively indicating fair to poor performance of the two tests. For anti-dengue IgG the performance of the test was fairly good unlike the IgM test kits which perform poorly. The Kappa values therefore suggested a fair RDT performance for IgG and poor for IgM hence were not used in the analysis of this study except to 
Table 5. Performance Characteristics of RDT.

\begin{tabular}{cccc}
\hline Antibody & Performance of RDT & Percentage & Kappa value \\
\hline \multirow{3}{*}{ IgG } & Sensitivity & $26.5 \%$ & 0.373 \\
& Specificity & $99.6 \%$ & \\
& Positive predictive value & $90 \%$ & \\
& Negative predictive value & $90.4 \%$ & 0.0001 \\
IgM & Sensitivity & 0 & \\
& Specificity & $100 \%$ & \\
& Positive predictive value & 0 & \\
\hline
\end{tabular}

show their seropositive and negative rate.

\section{Discussion}

Dengue is an important public health problem in the tropics and sub-tropics of the world and the prevalence is becoming high especially in sub-Saharan Africa [12]. In as much as knowing the global or national prevalence of a disease is important, it is also very essential to know the incidence of disease in a localized manner. In this study, the seroprevalence of IgG and IgM in local communities were $12.6 \%$ and $2.2 \%$ respectively. These findings were similar to and at the same time vary in other parts of the country. The seroprevalence in some of the regions of Ghana ranges from $3.6 \%$ to $57 \%$ in each case for anti-dengue IgG and IgM [13] [8]. The disparities between these seroprevalence values may be due to different geographical areas where these studies were carried out with different climatic conditions that might vary in favouring the existence of the virus vector. This further suggests the presence of the disease in the country but has been dormant in patients without any clinical conditions.

The variation in frequencies in the incidence of dengue by gender has been attributed to the differences in exposures to the virus such as time away from home [14]. Gender-related factors can also shift over the course of human lifespan and this will affect sexes in contrasting dengue viral infection. In this study, the seroprevalence was higher in females than males. This observation is in contrast with studies conducted in Agogo, Kintampo, Techiman and other parts of Ghana [7] [13]. Again, in India, [15] the seroprevalence was $24.9 \%$ among febrile patients of which males were twice more affected than females. A higher female than male seroprevalence was however reported in Nigeria [16] [17], which was also similar to this study. The variability in gender prevalence could be ascribed to exposure to Aedes aegypti due to occupational differences that might affect their exposure time to the mosquitoes. The mosquito vector is diurnal, endophilic and endophagic and therefore, high seroprevalence in female than male could be attributed to the fact that females were more likely to remain at home and engage in domestic activities during the day before dusk where the 
vector is most active. Again, the variation in gender seroprevalence could be attributed to sampling.

The presence of only immunoglobulin $M$ in individuals denotes a recent or primary infection. This implies that individuals are susceptible to a secondary heterologous infection by a different serotype but have a lifelong immunological protection against a homologous serotype. The implication of those with the presence of both IgM and IgG may suggest secondary acute heterologous infection but clinically healthy because they may not have risk factors such as age, host immune response and strain virulence. They were therefore likely not having comorbidities such as diabetes, bronchial asthma, fulminant hepatitis among others that may be implicated in dengue hemorrhagic fever. Individuals with past primary or probable secondary infection were those with IgG antibodies and may have protection against secondary homologous infection. Lastly, the group with no antibodies is likely to be susceptible to primary dengue infection by all serotypes because they have not built-up any antibodies against the virus [17].

The use of RDTs for serological testing is very common as a result of being very affordable, readily available and quick in its operation. However, there has been no proper regulations by national and even international testing agencies even though the market for RDTs continues to grow [18]. The sensitivity and specificity values of these kits corresponded with a study [19] which looked at the performance of some commercial RDTs for the diagnosis of dengue infection. Low to high sensitivity and specificity values varying from $6 \%$ to $65 \%$ and $69 \%$ to $100 \%$ respectively were shown indicating a varying specificity and sensitivity with the use of RDTs. Low sensitivities ranging from $27.8 \%$ to $77.7 \%$, however, with higher specificities ranging from $50 \%$ to $100 \%$ were found in some cases [19]. These results were distinctively different from the performance characteristics claimed by the manufacturers. It is therefore imperative to properly evaluate the RDT kits being sold in the market.

Elmina recorded the highest seroprevalence value for both anti-dengue IgG and IgM. This indicates that the virus is being transmitted among the population with improper sanitation. About $71.6 \%$ of households in the municipality use improper sanitation facilities [11]. Therefore, Elmina township needs sustainable sanitation service to match the increasing population. The transmission of dengue can display considerable geographical variability at a small spatial scale with significant variations in incidence in neighboring towns among urban settings [20]. Neighborhoods that were deprived of basic sanitary and hygienic conditions recorded a significantly high seropositive rate as compared to areas with good hygienic measures [20]. Again factors that account for differences in the transmission of dengue include level of urbanization, household risk factor, physical environment and vector ecology [21] [22]. Marshes and swamps have also been identified to play a high significant role in dengue vector growth [23]. It is worthy to note that the Ministry of Lands and Forestry (1999) and Asoma- 
nin (2019) have listed Elmina as one of the coastal areas in Ghana with wetlands. Some of this dengue transmission contributing factors might have accounted for the high seropositivity rate for both anti-dengue IgG and IgM in the Elmina township.

\section{Conclusion}

The seropositivity of IgG and IgM among participants suggests dengue virus may be in circulation at Cape Coast Metropolis and Komenda Edina Eguafo Abirem Municipality. Elmina showed the highest seroprevalence of IgG and IgM and may likely be a major area of the transmission of the virus to the other municipalities. This therefore requires further detailed research work to establish the presence of the virus in Ghana and the subsequent creation of dengue awareness, enhance outbreak preparedness and resource the hospital laboratories with diagnostic tools and equipment for the detection and management of the infection.

\section{Limitation of Study}

This study had a limitation. The flavivirus group of viruses has a similar genome hence can cross-react with each other. There was therefore the need to confirm samples that tested positive for IgG and IgM with a gold standard confirmatory test such as the plaque neutralization reduction test (PRNT); this was not done due to its unavailability.

\section{Conflicts of Interest}

The authors declare no conflicts of interest regarding the publication of this paper.

\section{References}

[1] World Health Organization (2012) Global Strategy for Dengue Prevention and Control 2012-2020. World Health Organization, Geneva, 43.

[2] Gubler, D.J. (2012) The Economic Burden of Dengue. American Journal of Tropical Medicine and Hygiene, 86, 743-744. https://doi.org/10.4269/ajtmh.2012.12-0157

[3] World Health Organization (2009) Dengue: Guidelines for Diagnosis, Treatment, Prevention, and Control. New Edition, World Health Organization, Geneva.

[4] World Health Organization (2006) Scientific Working Group United Nations Children's Fund/United Nations Development Programme/World Bank/World Health Organization.

[5] Brady, O.J., Gething, P.W., Bhatt, S., Messina, J.P., Brownstein, J.S., Hoen, A.G., et al. (2012) Refining the Global Spatial Limits of Dengue Virus Transmission by Evidence-Based Consensus. PLoS Neglected Tropical Diseases, 6, e1760. https://doi.org/10.1371/journal.pntd.0001760

[6] Stoler. J., al Dashti, R., Anto, F., Fobil, J.N. and Awandare, G.A. (2014) Deconstructing "Malaria": West Africa as the Next Front for Dengue Fever Surveillance and Control. Acta Tropica, 134, 58-65. 
https://doi.org/10.1016/j.actatropica.2014.02.017

[7] Narkwa, P.W., Mutocheluh, M., Kwofie, T.B., Owusu, M., Annan, A., Ali, I., et al. (2016) Dengue Virus Exposure among Blood Donors in Ghana. Journal of Medical and Biomedical Sciences, 5, 30-35.

[8] Humphrey, J.M., Cleton, N.B., Reusken, C.B.E.M., Glesby, M.J., Koopmans, M.P.G. and Abu-Raddad, L.J. (2016) Dengue in the Middle East and North Africa: A Systematic Review. PLoS Neglected Tropical Diseases, 10, e0005194. https://doi.org/10.1371/journal.pntd.0005194

[9] Stoler, J., Delimini, R.K., Kofi Bonney, J.H., Oduro, A.R., Owusu-Agyei, S., Fobil, J.N., et al. (2015) Evidence of Recent Dengue Exposure among Malaria Parasite-Positive Children in Three Urban Centers in Ghana. American Journal of Tropical Medicine and Hygiene, 92, 497-500. https://doi.org/10.4269/ajtmh.14-0678

[10] Ayukekbong, J.A., Oyero, O.G., Nnukwu, S.E., Mesumbe, H.N. and Fobisong, C.N. (2017) Value of Routine Dengue Diagnosis in Endemic Countries. World Journal of Virology, 6, 9-16. https://doi.org/10.5501/wjv.v6.i1.9

[11] Service, G.S. (2013) Regional Analytical Report. Ghana Statistical Service, Accra, 61-70.

[12] Goswami, L., Runumi, C. and Rasul, E.S. (2018) Seroprevalence of Dengue Infection in a Tertiary Care Hospital in Assam. International Journal of Medical and Dental Sciences, 7, Article No. 1582. https://doi.org/10.18311/ijmds/2018/18905

[13] Ofosu-Appiah, L., Kutame, R., Ayensu, B., Bonney, J.H.K., Boateng, G., Bempong Adade, R., et al. (2018) Detection of Dengue Virus in Samples from Suspected Yellow Fever Cases in Ghana. Microbiology Research Journal International, 24, 1-10. https://doi.org/10.9734/MRJI/2018/41090

[14] Anker, M. and Arima, Y. (2011) Male-Female Differences in the Number of Reported Incident Dengue Fever Cases in Six Asian Countries. Western Pacific Surveillance and Response, 2, 17-23.

[15] Chitkara, S., Chhina, D., Gupta, V., Mahajan, R. and Sharma, D. (2018) Epidemiology of Dengue Fever among clinically Suspected Febrile Patients at A Tertiary Care Center in Punjab. Journal of Microbiology and Infectious Diseases, 8, 43-48. https://doi.org/10.5799/jmid.434590

[16] Bello, O.A., Aminu, M. and Jatau, E.D. (2016) Seroprevalence of IgM Antibodies to Dengue Fever Virus among Patients Presenting with Symptoms of Fever in Some Hospitals in Kaduna State, Nigeria. International Journal of Science and Research, 5, 1255-1259. https://doi.org/10.21275/v5i3.NOV162015

[17] Sule, W.F., Fadamitan, T.O., Lawal, O.A., Adebimpe, W.O., Opaleye, O.O. and Oluwayelu, D.O. (2019) Probable Primary and Secondary Dengue Viral Infections and Associated Host Factors among University Undergraduates in Osun State, Nigeria. Alexandria Journal of Medicine, 55, 25-30. https://doi.org/10.1080/20905068.2019.1592935

[18] Garg, A., Garg, J., Singh, D.V. and Dhole, T.N. (2019) Can Rapid Dengue Diagnostic Kits Be Trusted ? A Comparative Study of Commercially Available Rapid Kits for Serodiagnosis of Dengue Fever. Journal of Laboratory Physicians, 11, 63-67. https://doi.org/10.4103/JLP.JLP $140 \quad 18$

[19] Blacksell, S.D., Newton, P.N., Bell, D., Kelley, J., Mammen Jr., M.P., Vaughn, D.W., et al. (2006) The Comparative Accuracy of 8 Commercial Rapid Immunochromatographic Assays for the Diagnosis of Acute Dengue Virus Infection. Clinical Infectious Diseases, 42, 1127-1134. https://doi.org/10.1086/501358 
[20] Braga, C., Feitosa Luna, C., Martelli, C.M.T., de Souza, W.V., Tenório Cordeiro, M., Alexander, N., et al. (2010) Seroprevalence and Risk Factors for Dengue Infection in Socio-Economically Distinct Areas of Recife, Brazil. Acta Tropica, 113, 234-240. https://doi.org/10.1016/j.actatropica.2009.10.021

[21] Restrepo, A.C., Baker, P. and Clements, A.C.A. (2014) National Spatial and Temporal Patterns of Notified Dengue Cases, Colombia 2007-2010. Tropical Medicine \& International Health, 19, 863-871. https://doi.org/10.1111/tmi.12325

[22] Vanwambeke, S.O., van Benthem, B.H.B., Khantikul, N., Burghoorn-Maas, C., Panart, K., Oskam, L., et al. (2006) Multi-Level Analyses of Spatial and Temporal Determinants for Dengue Infection. International Journal of Health Geographics volume, 5, Article No. 5. https://doi.org/10.1186/1476-072X-5-5

[23] Sarfraz, M.S., Tripathi, N.K., Tipdecho, T., Thongbu, T., Kerdthong, P. and Souris, M. (2012) Analyzing the Spatio-Temporal Relationship between Dengue Vector Larval Density and Land-Use Using Factor Analysis and Spatial Ring Mapping. BMC Public Health, 12, Article No. 853. https://doi.org/10.1186/1471-2458-12-853 